\title{
DECOMPOSIÇÃO MODAL DE LINHAS DE TRANSMISSÃO A PARTIR DO USO DE DUAS MATRIZES DE TRANSFORMAÇÃO
}

\author{
Sérgio Kurokawa* \\ kurokawa@dee.feis. unesp.br \\ Afonso José do Prado* \\ afonsojp@dee.feis.unesp.br \\ Rodrigo Serra Daltin* \\ Rodrigo_daltinesuperig.com.br \\ Luiz Fernando Bovolato* \\ bovolato@dee.feis.unesp.br \\ José Pissolato ${ }^{\dagger}$ \\ pissoddsce.fee.unicamp.br \\ * Departamento de Engenharia Elétrica, \\ Faculdade de Engenharia de Ilha Solteira, UNESP, CP 31, CEP 15385-000, \\ Ilha Solteira, SP, Brasil \\ ${ }^{\dagger}$ Departamento de Sistemas e Controle de Energia, \\ Faculdade de Engenharia Elétrica e de Computação, UNICAMP, CP 6101, CEP 13081-970, \\ Campinas, SP, Brasil
}

\section{RESUMO}

Este artigo mostra um processo alternativo de decomposição modal de linhas de transmissão trifásicas, não idealmente transpostas, que possuem um plano de simetria vertical. O processo baseia-se no uso de duas matrizes de transformação modal. Inicialmente utiliza-se a matriz de Clarke para decompor a linha em suas componentes $\alpha, \beta$ e zero. Devido ao fato de as componentes $\alpha$ e zero serem acopladas, as mesmas podem ser representadas como sendo uma linha bifásica sem um plano de simetria vertical. Em seguida esta linha bifásica é decomposta em seus modos exatos a partir do uso de uma matriz de decomposição modal adequada. $\mathrm{O}$ artigo descreve as características da linha bifásica que representa as componentes $\alpha$ e zero e mostra a aplicação do processo de decomposição modal em uma linha trifásica de $440 \mathrm{kV}$.

PALAVRAS-CHAVE: Transitórios eletromagnéticos, domínio da frequiência, análise modal, linha de transmissão.

\section{ABSTRACT}

This paper shows an alternative modal decomposition procedure of non ideally transposed three-phase transmission lines with a vertical symmetry. The procedure is based in the use of two modal transformation matrices. First, Clarke's matrix is used to separate the line in its $\alpha, \beta$ and zero components. Because $\alpha$ and zero components are mutually coupled they can be represented as being a two-phase line without a vertical symmetry plane. After that this two-phase line is separated in its exact modes by using an adequated modal transformation matrix. The paper describes the characteristics of the two-phase line used to represent the coupled $\alpha$ and zero components and it shows the application of the modal decomposition procedure in a $440 \mathrm{kV}$ three-phase transmission line.

KEYWORDS: Electromagnetic transients, frequency domain, modal analysis, transmission line.

\footnotetext{
Artigo submetido em 08/06/2006

1a. Revisão em 08/12/2006

2a. Revisão em 27/03/2007

Aceito sob recomendação do Editor Associado

Prof. Denis Vinicius Coury
} 


\section{INTRODUÇÃO}

A linha de transmissão, em análises e estudos de transitórios eletromagnéticos em sistemas de potência, pode ser representada no domínio das fases ou no domínio modal.

No domínio modal, uma linha polifásica de $n$ fases pode ser separada em seus $n$ modos de propagação e cada um destes modos comporta-se como uma linha monofásica. Sendo assim, pode-se dizer que no domínio modal uma linha de $n$ fases pode ser representada por $n$ linhas monofásicas, sendo que cada uma destas linhas é independente das demais.

A vantagem da representação no domínio modal é que neste domínio o acoplamento entre as fases da linha é eliminado, tornando mais fácil a obtenção da solução das equações diferenciais que descrevem o comportamento das correntes e tensões ao longo da linha.

A decomposição de uma linha de transmissão em seus modos de propagação é feita por meio do uso de uma matriz de transformação $\left[\mathrm{T}_{I}\right]$ cujas colunas são autovetores associados aos autovalores do produto [Y][Z], sendo que [Z] é a matriz de impedância longitudinal e [Y] é a matriz de admitância transversal da linha (Wedepohl et alii, 1996).

Os elementos da matriz $\left[\mathrm{T}_{I}\right]$ geralmente são elementos pertencentes ao conjunto dos números complexos e são também variáveis em função da freqüência, sendo que a dependência em relação à freqüência torna difícil a representação desta matriz diretamente no domínio do tempo (Tavares et alii, 1998).

No entanto, existem linhas de transmissão cuja configuração geométrica permite que os elementos da matriz $\left[\mathrm{T}_{I}\right]$ sejam reais, independentes da frequiência e, conseqüentemente, facilmente representados no domínio do tempo. Como exemplo de linhas cuja matriz $\left[\mathrm{T}_{I}\right]$ possuem somente elementos reais e constantes, pode-se mencionar as linhas bifásica com plano de simetria vertical (Budner, 1970) e as linhas trifásicas idealmente transpostas (Tavares et alii, 1999).

No caso de linhas trifásicas que não podem ser consideradas idealmente transpostas mas que possuem um plano de simetria vertical, a matriz de Clarke separa a mesma nas componentes $\alpha, \beta$ e zero. A componente $\beta$ é um modo exato enquanto que as componentes $\alpha$ e zero são acopladas mutuamente e são denominados quase-modos. No entanto, existem situações bem restritas em que o acoplamento entre as componentes $\alpha$ e zero pode ser desconsiderado, fazendo com que estas componentes tornem-se também modos exatos da linha. Nestas condições, diz-se que a linha trifásica não idealmente transposta mas que possui um plano de simetria vertical pode ser decomposta em seus modos de propagação por meio do uso da matriz de Clarke, que é real, independente da freqüên- cia e facilmente representada no domínio do tempo (Tavares et alii, 1999).

Para as situações em que o uso da matriz de Clarke como sendo uma matriz de transformação modal de linhas trifásicas, não idealmente transpostas que possuem um plano de simetria vertical, este trabalho propõe utilizar duas matrizes de transformação para decompor a linha em seus modos exatos. Inicialmente será utilizada a matriz de Clarke para decompor a linha em suas componentes $\alpha, \beta$ e zero. Portanto a linha trifásica pode ser representada por uma linha monofásica (componente $\beta$ ) e por uma linha bifásica (componentes $\alpha$ e zero), sendo que não existe acoplamento entre estas duas linhas. A linha bifásica, que representa as componentes $\alpha$ e zero, pode então ser representada no domínio modal por meio do uso de uma matriz de transformação adequada.

O método proposto será aplicado na decomposição modal de uma linha trifásica de $440 \mathrm{kV}$, não transposta e que possui um plano de simetria vertical.

\section{REPRESENTAÇÃO NO DOMÍNIO MO- DAL}

Sabe-se que as correntes e tensões ao longo do comprimento de uma linha de transmissão polifásica de $n$ fases podem ser descritas a partir da solução das seguintes equações diferenciais (Budner, 1970):

$$
\begin{aligned}
& \frac{d^{2}\left[V_{f}\right]}{d x^{2}}=[Z][Y]\left[V_{f}\right] \\
& \frac{d^{2}\left[I_{f}\right]}{d x^{2}}=[Y][Z]\left[I_{f}\right]
\end{aligned}
$$

Nas equações (1) e (2) as matrizes [Z] e [Y] são, respectivamente, as matrizes de impedâncias longitudinais e de admitâncias transversais por unidade de comprimento da linha. Os vetores $\left[\mathrm{V}_{f}\right]$ e $\left[\mathrm{I}_{f}\right]$ contém, respectivamente, as tensões e correntes de fase da linha.

Diz-se que as equações (1) e (2) são as equações diferenciais da linha no domínio das fases. Estas equações diferenciais são de difícil solução, uma vez que os produtos matriciais $[\mathrm{Z}][\mathrm{Y}]$ e $[\mathrm{Y}][\mathrm{Z}]$ resultam em matrizes cheias, geralmente distintas.

No entanto pode-se utilizar uma transformação de similaridade (Chen, 1984) e transformar os produtos [Z][Y] e [Y][Z] em matrizes diagonais. Nestas condições, a solução das equações diferenciais da linha podem ser obtidas mais facilmente.

Considerando uma matriz $\left[\mathrm{T}_{I}\right]$ cujas colunas são autovetores 
associados aos autovalores do produto matricial [Y][Z] podese escrever (Wedepohl et alii, 1996):

$$
[\lambda]=\left[T_{I}\right]^{-1}[Y][Z]\left[T_{I}\right]
$$

Na equação (3) $[\lambda]$ é uma matriz diagonal cujos elementos da diagonal principal são os autovalores do produto matricial $[\mathrm{Y}][\mathrm{Z}]$.

Manipulando a equação (3) e substituindo-a nas equações (1) e (2) pode-se obter:

$$
\begin{gathered}
\frac{d^{2}\left[E_{m}\right]}{d x^{2}}=[\lambda]\left[E_{m}\right] \\
\frac{d^{2}\left[I_{m}\right]}{d x^{2}}=[\lambda]\left[I_{m}\right]
\end{gathered}
$$

sendo

$$
\begin{aligned}
& {\left[E_{m}\right]=\left[T_{I}\right]^{T}\left[V_{f}\right]} \\
& {\left[I_{m}\right]=\left[T_{I}\right]^{-1}\left[I_{f}\right]}
\end{aligned}
$$

As equações (4) e (5) são as equações diferenciais da linha no domínio modal. Diz-se que a linha polifásica de $n$ fases foi decomposta em seus $n$ modos de propagação.

Nas equações (4)-(7) $\left[E_{m}\right]$ e $\left[I_{m}\right]$ são respectivamente, os vetores com as tensões transversais e as correntes longitudinais ao longo de cada modo da linha. A matriz $\left[\mathrm{T}_{I}\right]^{T}$ é a transposta de $\left[\mathrm{T}_{I}\right]$ e $\left[\mathrm{T}_{I}\right]^{-1}$ é a inversa de $\left[\mathrm{T}_{I}\right]$.

Devido ao fato de que $[\lambda]$ é uma matriz diagonal, podese afirmar que no domínio modal uma linha de $n$ fases comporta-se como sendo $n$ linhas monofásicas desacopladas, sendo que as soluções das equações diferenciais de linhas monofásicas são conhecidas (Budner, 1970).

Mostra-se também que é possível escrever as seguintes relações entre as grandezas de fase e de modo (Wedepohl et alii, 1996):

$$
\begin{gathered}
{\left[Z_{m}\right]=\left[T_{I}\right]^{T}[Z]\left[T_{I}\right]} \\
{\left[Y_{m}\right]=\left[T_{I}\right]^{-1}[Y]\left[T_{I}\right]^{-T}}
\end{gathered}
$$

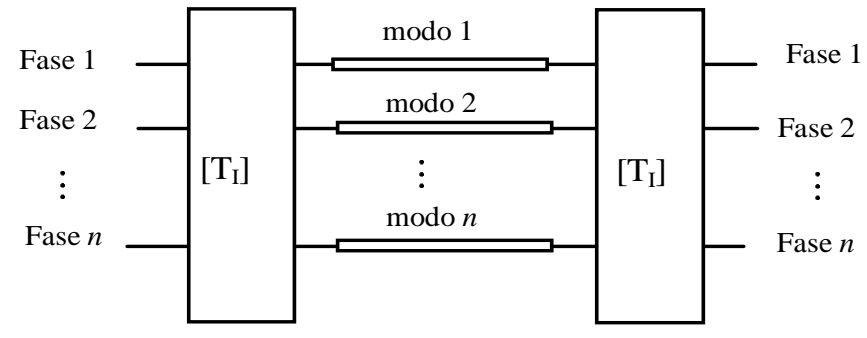

Figura 1: Representação modal de uma linha polifásica.

Nas equações (8) e (9) $\left[\mathrm{Z}_{m}\right]$ e $\left[\mathrm{Y}_{m}\right]$ são matrizes diagonais (Wedepohl et alii, 1996) que contém, respectivamente, as impedâncias e admitâncias modais por unidade de comprimento da linha. A matriz $\left[\mathrm{T}_{I}\right]^{-T}$ é a inversa de $\left[\mathrm{T}_{I}\right]^{T}$.

Deste modo, um linha polifásica de $n$ fases pode ser representada no domínio modal, onde cada modo comporta-se como uma linha monofásica. No domínio modal é possível calcular as correntes e tensões em cada um dos $n$ modos. Em seguida, pode-se converter as correntes e tensões para o domínio das fases.

A Figura 1 ilustra a representação de uma linha de transmisão de $\mathrm{n}$ fases no domínio modal.

Na Figura 1 as grandezas de fase são convertidas para grandezas modais através da matriz de transformação modal $\left[\mathrm{T}_{I}\right]$. Em seguida, as correntes e tensões em cada um dos modos da linha são calculadas, levando-se em consideração que cada um destes modos comporta-se como uma linha monofásica sem nenhum acoplamento com os demais modos. Em uma outra etapa as correntes e tensões são convertidas do domínio modal para o domínio das fases.

\section{UTILIZAÇÃO DA MATRIZ DE CLARKE}

Considere uma linha de transmissão trifásica não idealmente transposta, mas que possui um plano de simetria vertical, conforme mostra a Figura 2.

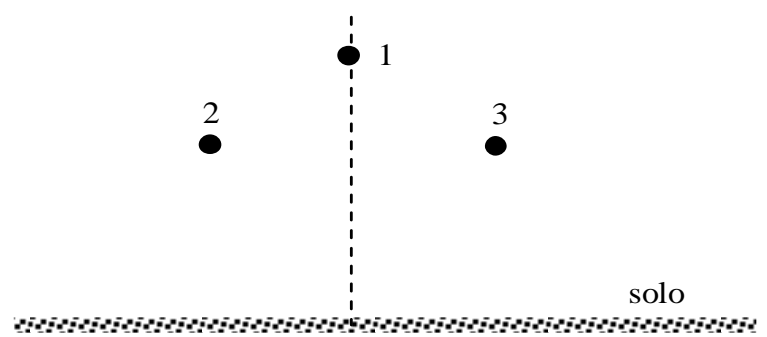

Figura 2: Silhueta de uma linha trifásica com plano de simetria vertical. 
As matriz de impedância longitudinal [Z] desta linha apresenta a seguinte configuração (Tavares et alii, 1999):

$$
[Z]=\left[\begin{array}{lll}
A & D & D \\
D & B & F \\
D & F & B
\end{array}\right]
$$

A matriz de admitância [Y] tem a mesma estrutura da matriz [Z].

Devido ao fato de que a linha trifásica mostrada na Figura 2 possui um plano de simetria vertical, a mesma pode ser decomposta em seus quase-modos a partir do uso da matriz de Clarke como sendo uma matriz de transformação modal (Tavares et alii, 1999). Substituindo a matriz $\left[\mathrm{T}_{I}\right]$ pela matriz de Clarke nas equações (8) e (9), as mesmas pasam a ser escritas como sendo:

$$
\begin{gathered}
{\left[Z_{\alpha \beta 0}\right]=\left[T_{\text {Clarke }}\right]^{T}[Z]\left[T_{\text {Clarke }}\right]} \\
{\left[Y_{\alpha \beta 0}\right]=\left[T_{\text {Clarke }}\right]^{-1}[Y]\left[T_{\text {Clarke }}\right]^{-T}}
\end{gathered}
$$

Nas equações (11) e (12) [ $\left.\mathrm{T}_{\text {clarke }}\right]$ é a matriz de Clarke e é escrita como sendo:

$$
\left[T_{\text {Clarke }}\right]=\left[\begin{array}{ccc}
\frac{2}{\sqrt{6}} & 0 & \frac{1}{\sqrt{3}} \\
-\frac{1}{\sqrt{6}} & \frac{1}{\sqrt{2}} & \frac{1}{\sqrt{3}} \\
-\frac{1}{\sqrt{6}} & -\frac{1}{\sqrt{2}} & \frac{1}{\sqrt{3}}
\end{array}\right]
$$

Desenvolvendo as equações (11) e (12) obtém-se (Tavares et alii, 1999):

$$
\begin{aligned}
& {\left[Z_{\alpha \beta 0}\right]=\left[\begin{array}{ccc}
z_{\alpha} & 0 & z_{\alpha 0} \\
0 & z_{\beta} & 0 \\
z_{\alpha 0} & 0 & z_{0}
\end{array}\right]} \\
& {\left[Y_{\alpha \beta 0}\right]=\left[\begin{array}{ccc}
y_{\alpha} & 0 & y_{\alpha 0} \\
0 & y_{\beta} & 0 \\
y_{\alpha 0} & 0 & y_{0}
\end{array}\right]}
\end{aligned}
$$

Observa-se que se a matriz de Clarke é utilizada como sendo uma matriz de transformação modal, a linha trifásica mostrada na Figura 2 é separada em três componentes. As componentes $\alpha$ e zero são acopladas entre si enquanto que a componente $\beta$ é totalmente desacoplada em relação às componentes $\alpha$ e zero. Devido ao acoplamento mútuo, as componentes $\alpha$ e zero são denominadas quase-modos da linha e a componente $\beta$, por ser totalmente desacoplada, é um modo exato da linha.

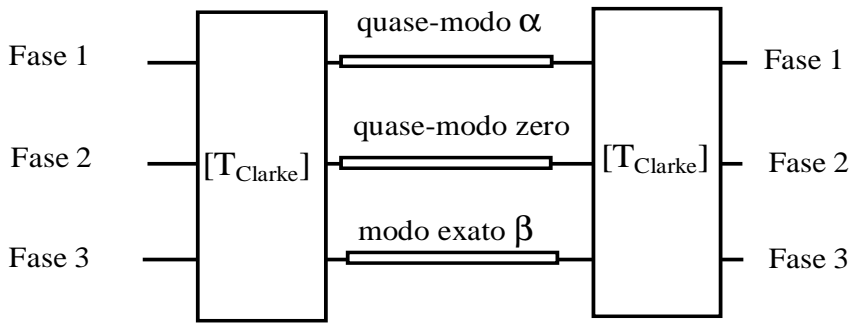

Figura 3: Decomposição modal com a matriz de Clarke.

Há situações bem particulares (tais como em simulações de transitórios decorrentes de operações de manobras e chaveamentos de linhas) em que os termos mútuos $\mathrm{z}_{\alpha 0}$ e $\mathrm{y}_{\alpha 0}$ podem ser desprezados. Nestas condições, as matrizes $\left[\mathrm{Z}_{\alpha \beta 0}\right]$ e [ $\left.\mathrm{Y}_{\alpha \beta 0}\right]$ são escritas como sendo:

$$
\begin{aligned}
& {\left[\mathrm{Z}_{\alpha \beta 0}\right] \cong\left[\begin{array}{ccc}
z_{\alpha} & 0 & 0 \\
0 & z_{\beta} & 0 \\
0 & 0 & z_{0}
\end{array}\right]} \\
& {\left[\mathrm{Y}_{\alpha \beta 0}\right] \cong\left[\begin{array}{ccc}
y_{\alpha} & 0 & 0 \\
0 & y_{\beta} & 0 \\
0 & 0 & y_{0}
\end{array}\right]}
\end{aligned}
$$

Observa-se que se o acoplamento entre os quase-modos $\alpha \mathrm{e}$ zero é desprezado, a matriz de Clarke separa a linha trifásica mostrada na Figura 1 em seus modos exatos.

A Figura 3 mostra a representação esquemática da decomposição modal de uma linha trifásica considerando-se a matriz de Clarke como sendo a matriz de decomposição modal.

Devido ao fato da matriz de Clarke ser uma matriz real e independente da freqüência, a mesma pode ser facilmente implementada em programas que realizam simulações de transitórios eletromagnéticos diretamente no domínio do tempo.

\section{UTILIZAÇÃO DE DUAS MATRIZES}

\subsection{Descrição Geral}

Em situações em que a matriz de Clarke não pode ser considerada como sendo a matriz de transformação modal da linha cuja silhueta é mostrada na Figura 2, este artigo propõe o uso de duas matrizes de transformação para obter os modos exatos da linha. Inicialmente utiliza-se a matriz de Clarke para obter as componentes $\alpha, \beta$ e zero. Em seguida os quase-modos $\alpha$ e zero são considerados como sendo uma linha bifásica sem plano de simetria vertical, que é então separada em seus dois modos exatos por meio do uso de uma matriz de decomposição modal $\left[\mathrm{T}_{\alpha 0}\right]$ adequada. Se os dois 


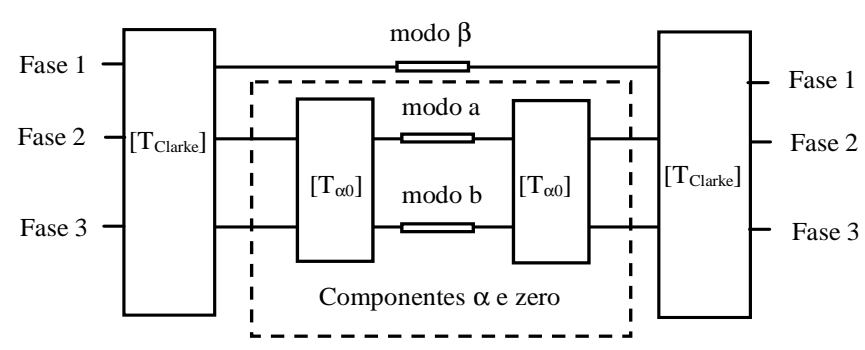

Figura 4: Decomposição modal utilizando duas matrizes de transformação.

modos a e b das componentes $\alpha$ e zero tiverem as características de linhas monofásicas, os mesmos poderão então ser representados por meio de modelos adequados de linhas.

A Figura 4 mostra a representação esquemática da decomposição modal de uma linha trifásica que possui um plano de simetria vertical, por meio do uso de duas matrizes de transformação

Na Figura $4\left[\mathrm{~T}_{\alpha o}\right]$ é a matriz que separa a linha bifásica (que representa os quase-modos $\alpha$ e zero) em seus modos exatos. Esta matriz, cujas colunas são autovetores associados aos autovalores do produto matricial $\left[\mathrm{Z}_{\alpha \beta 0}\right]\left[\mathrm{Y}_{\alpha \beta 0}\right]$, pode ser obtida por meio de métodos analíticos (Chen, 1984) ou numéricos (Wedepohl et alii, 1996).

Independentemente do método de cálculo utilizado para obter os autovetores, recomenda-se impor uma condição que garanta que os mesmos possuam módulo unitário. Esta condição garante que os autovetores sejam contínuos e não sofram variações abruptas em uma ampla faixa de frequiências. Autovetores com estas características são mais facilmente representados por meio de funções racionais (Wedepohl et alii, 1996), sendo esta representação de importância fundamental quando as simulações são realizadas diretamente no domínio do tempo (Sarto et alii, 2001).

\subsection{Decomposição Modal dos Quase- modos $\alpha$ e Zero}

Se a componente $\beta$ for excluída das matrizes $\left[\mathrm{Z}_{\alpha \beta 0}\right]$ e $\left[\mathrm{Y}_{\alpha \beta 0}\right]$ as mesmas tornam-se:

$$
\begin{aligned}
& {\left[\mathrm{Z}_{\alpha 0}\right]=\left[\begin{array}{cc}
z_{\alpha} & z_{\alpha 0} \\
z_{\alpha 0} & z_{0}
\end{array}\right]} \\
& {\left[\mathrm{Y}_{\alpha 0}\right]=\left[\begin{array}{cc}
y_{\alpha} & y_{\alpha 0} \\
y_{\alpha 0} & y_{0}
\end{array}\right]}
\end{aligned}
$$

As matrizes $\left[\mathrm{Z}_{\alpha 0}\right]$ e $\left[\mathrm{Y}_{\alpha 0}\right]$, mostrada anteriormente, possuem

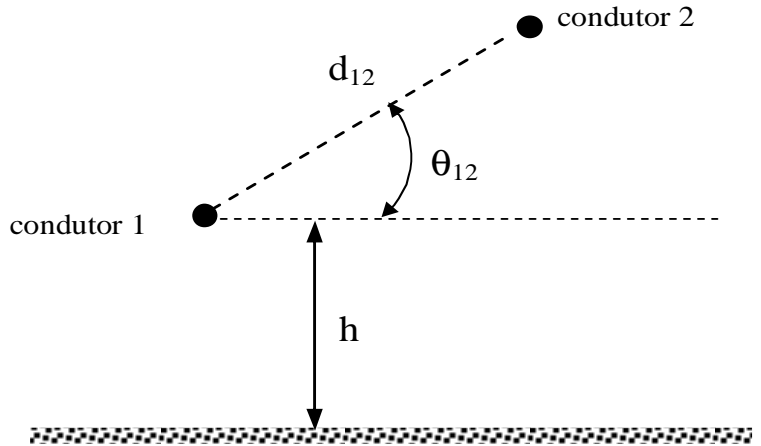

Figura 5: Linha bifásica descrita por $\left[\mathrm{Z}_{\alpha 0}\right]$ e $\left[\mathrm{Y}_{\alpha 0}\right]$.

as características das matrizes de impedância longitudinal e de admitância transversal, respectivamente, de uma linha bifásica sem plano de simetria vertical do tipo mostrada na Figura 5.

Na figura 5, os condutores 1 e 2 são as fases da linha bifásica que representa as componentes $\alpha$ e zero. $\mathrm{O}$ acoplamento entre as componentes $\alpha$ e zero é representado pelo acoplamento entre os condutores 1 e 2 .

$\mathrm{O}$ condutor 1 encontra-se a uma altura genérica h. Na mesma figura, $\mathrm{d}_{12}$ é a distância genérica entre os condutores 1 e 2 e $\theta_{12}$ pode assumir quaisquer valores desde que $\theta_{12} \neq 0$ e $\theta_{12} \neq \pi$.

As matrizes de impedância e de admitância modais da linha, mostrada na Figura 5, podem ser obtidas por meio das equações (8) e (9) e são escritas como sendo:

$$
\begin{gathered}
{\left[\mathrm{Z}^{\prime}\right]=\left[\mathrm{T}_{\alpha 0}\right]^{\mathrm{T}}\left[\mathrm{Z}_{\alpha 0}\right]\left[\mathrm{T}_{\alpha 0}\right]} \\
{\left[\mathrm{Y}^{\prime}\right]=\left[\mathrm{T}_{\alpha 0}\right]^{-1}\left[\mathrm{Y}_{\alpha 0}\right]\left[\mathrm{T}_{\alpha 0}\right]^{-\mathrm{T}}}
\end{gathered}
$$

Nas equações (20) e (21) [ $\left.\mathrm{T}_{\alpha o}\right]$ é uma matriz cujas colunas são os autovetores do produto $\left[\mathrm{Y}_{\alpha o}\right]\left[\mathrm{Z}_{\alpha o}\right]$. As matrizes [Z'] e [Y'] são, respectivamente, as matrizes de impedância longitudinal e de admitância transversal da linha no domínio modal e são escritas como sendo:

$$
\begin{aligned}
& {\left[\mathrm{Z}^{\prime}\right]=\left[\begin{array}{cc}
z_{a} & 0 \\
0 & z_{b}
\end{array}\right]} \\
& {\left[\mathrm{Y}^{\prime}\right]=\left[\begin{array}{cc}
y_{a} & 0 \\
0 & y_{b}
\end{array}\right]}
\end{aligned}
$$

Observa-se que as componentes $\alpha$ e zero, que são obtidas a partir do uso da matriz de Clarke como sendo uma matriz 
de transformação modal da linha trifásica, são separadas em duas componentes desacopladas a e b.

Para que estas componentes a e b possam ser consideradas linhas monofásicas, os elementos $\mathrm{z}_{a}, \mathrm{z}_{b}, \mathrm{y}_{a}$ e $\mathrm{y}_{b}$ devem possuir um comportamento tal que permita que os mesmos possam ser escritos na forma:

$$
\begin{aligned}
z_{a} & =R_{a}+j X_{a} \\
z_{b} & =R_{b}+j X_{b} \\
y_{a} & =G_{a}+j B_{a} \\
y_{b} & =G_{b}+j B_{b}
\end{aligned}
$$

Nas equações (24)-(27) os termos R, X e B devem ser maiores que zero enquanto que o termo $G$ deve ser maior ou igual a zero. Nestas condições, os modos a e b podem ser considerados como sendo linhas monofásicas. Estas linhas monofásicas a e b são então caracterizadas por impedâncias longitudinais são $\mathrm{z}_{a}$ e $\mathrm{z}_{b}$, respectivamente, e por admitâncias transversais $\mathrm{y}_{a}$ e $\mathrm{y}_{b}$.

A utilização do método de decomposição modal proposto neste artigo pode simplificar bastante o processo de busca de uma matriz de transformação modal adequada para decompor linhas trifásicas não idealmente transpostas, mas que possuem um plano de simetria vertical, em seus modos exatos.

Esta simplificação está no fato de que uma linha trifásica, cujas fases são acopladas, é separada em uma linha monofásica e em uma linha bifásica, sendo que estas duas linhas não possuem acoplamento entre elas. Portanto, devemos nos preocupar somente com o acoplamento entre as fases da linha bifásica. Acreditamos que esta é amaior contribuição do artigo.

Novos estudos devem ser desenvolvidos para encontrar uma matriz de decomposição modal adequada para a linha bifásica mostrada na Figura 5.

\section{APLICAÇÃO DO MÉTODO}

A Figura 6 mostra a silhueta de uma linha trifásica de $440 \mathrm{kV}$, não idealmente transposta e que possui um plano de simetria vertical.

Na Figura 6, as fases 1, 2 e 3 são constituídas de 4 subcondutores do tipo Grosbeak. Os condutores 4 e 5 são cabos pára-raios são do tipo EHSW-3/8".

Os parâmetros longitudinais da linha mostrada na Figura 6 foram calculados levando em consideração os efeitos solo e pelicular (Dommel, 1986; Marti, 1983).

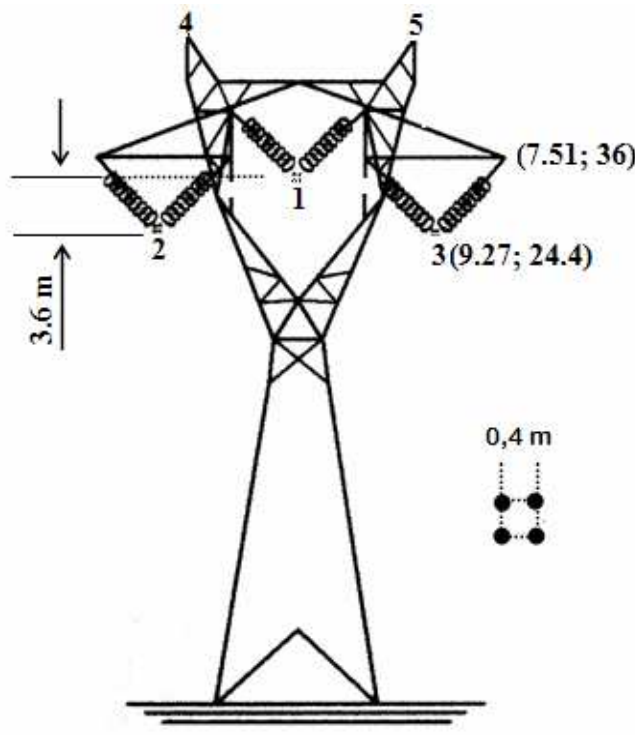

Figura 6: Silhueta de uma linha trifásica de $440 \mathrm{kV}$.

Para validar o processo de decomposição modal proposto neste artigo, o mesmo foi aplicado na linha mostrada na Figura 6 .

\subsection{Obtenção dos modos a e b}

Inicialmente a linha foi decomposta em suas componentes $\alpha, \beta$ e zero utilizando as equações (11) e (12). Em seguida, foi calculada uma matriz de transformação modal $\left[\mathrm{T}_{\alpha o}\right]$ que separa os quase modos $\alpha$ e zero em seus modos exatos a e b.

As Figuras 7 e 8 mostram as partes reais e imaginárias, respectivamente, dos elementos da matriz [Z'] da linha mostrada na Figura 6.

As figuras 7 e 8 mostram que os elementos da matriz [Z'] possuem partes real e imaginária positivas. Deste modo conclui-se que $\mathrm{z}_{a}$ e $\mathrm{z}_{b}$ comportam-se como impedâncias longitudinais de duas linhas monofásicas desacopladas.

As Figuras 9 e 10 mostram as partes reais e imaginárias, respectivamente, dos elementos da matriz [Y'].

As Figuras 9 e 10 mostram que os elementos da matriz [Y'] apresentam componentes reais praticamente nulas e partes imaginárias positivas. Portanto conclui-se que $\mathrm{y}_{a}$ e $\mathrm{y}_{b}$ comportam-se como admitâncias transversais de duas linhas monofásicas desacopladas.

Com base no comportamento dos elementos das matrizes [Z'] e [Y'], mostrados nas Figuras 7-10, conclui-se que os modos a e b comportam-se como duas linhas monofásicas independentes uma da outra. Estas linhas podem ser repre- 


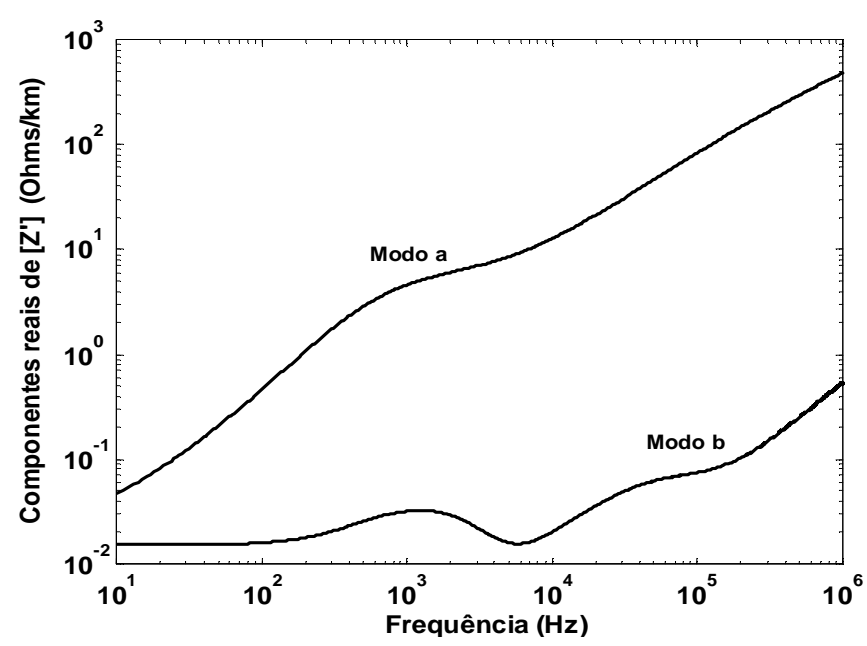

Figura 7: Parte real dos elementos da matriz [Z'].

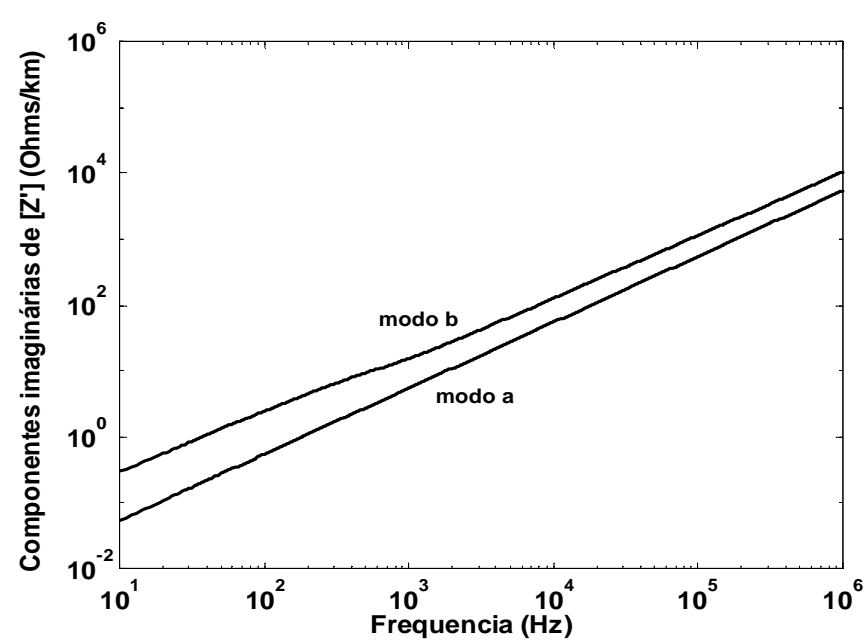

Figura 8: Parte imaginária dos elementos da matriz [Z’].

sentadas por meio de modelos adequados.

\subsection{Comparações}

Para comprovar que a representação modal proposta nete artigo está correta, a linha mostrada na Figura 6 foi representada por no domínio modal considerando duas situações distintas. Na primeira situação a linha foi decomposta em seus modos exatos por meio das expressões (3), (8) e (9) e, em uma segunda situação os modos exatos da linha foram obtidos por meio do método proposto neste artigo.

As duas representações modais, mencionadas anteriormente, foram utilizadas para obter a resposta em freqüência da linha mostrada na Figura 6. Neste teste uma fonte de tensão constante de 1 p.u foi aplicada na fase 1 da linha, conforme mostra

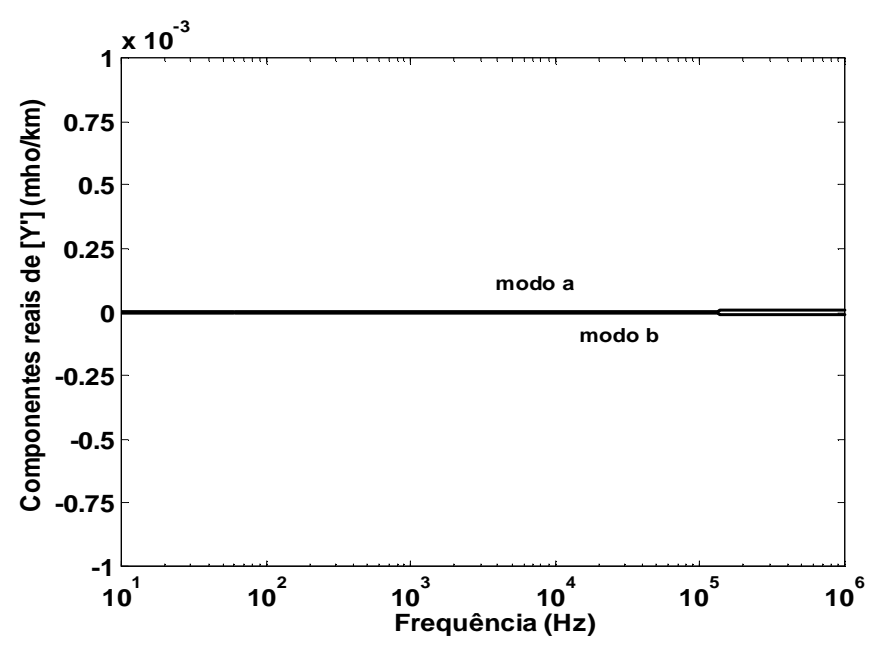

Figura 9: Parte real dos elementos da matriz [Y'].

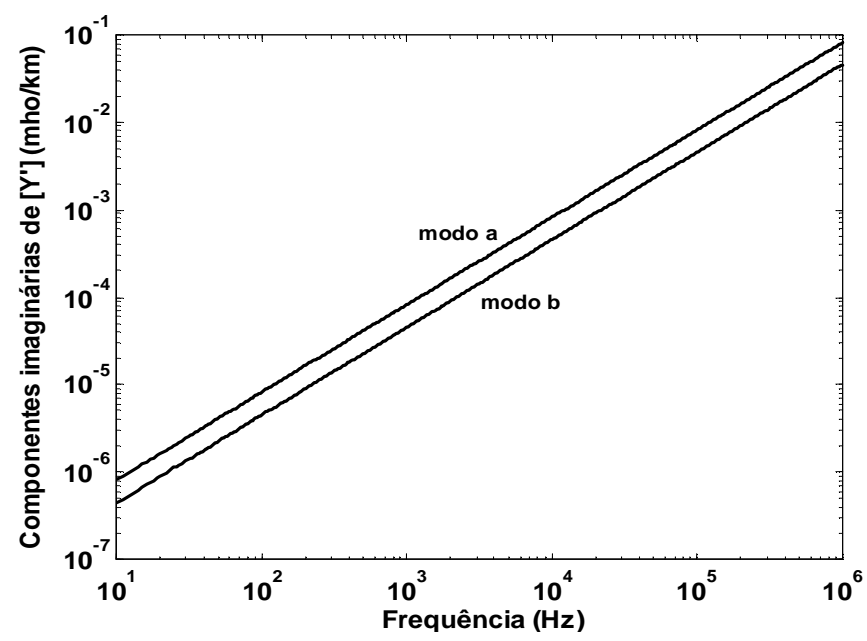

Figura 10: Parte imaginária dos elementos da matriz [Y'].

a Figura 11, enquanto que os terminais emissores das fases 2 e 3 foram mantidos aterrados. Considerou-se que a linha está conectada a uma carga idêntica à impedância característica Zc da linha, de modo a evitar que ocorram reflexões de correntes e tensões nos terminais da linha (Minegishi et alii, 1994).

Cada um dos modos da linha foi representado como sendo uma linha monofásica e as correntes e tensões modais foram calculadas por meio das soluções analíticas das equações diferenciais que caracterizam uma linha monofásica (Budner, 1970).

As Figuras 12 e 13 mostram o módulo das tensões nos terminais receptores das fases 1 e 2, respectivamente. A curva 1 mostra resultados obtidos quando a linha é decomposta em seus modos exatos por meio das expressões (3), (8) e (9), 


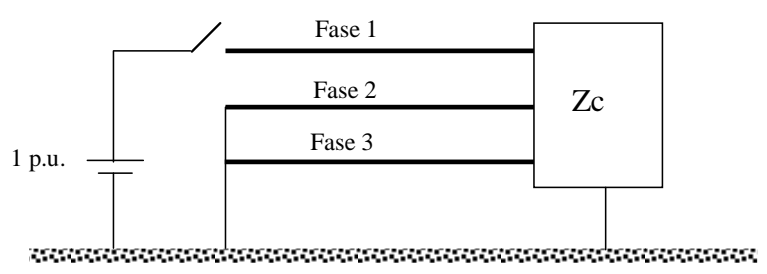

Figura 11: Energização da linha.

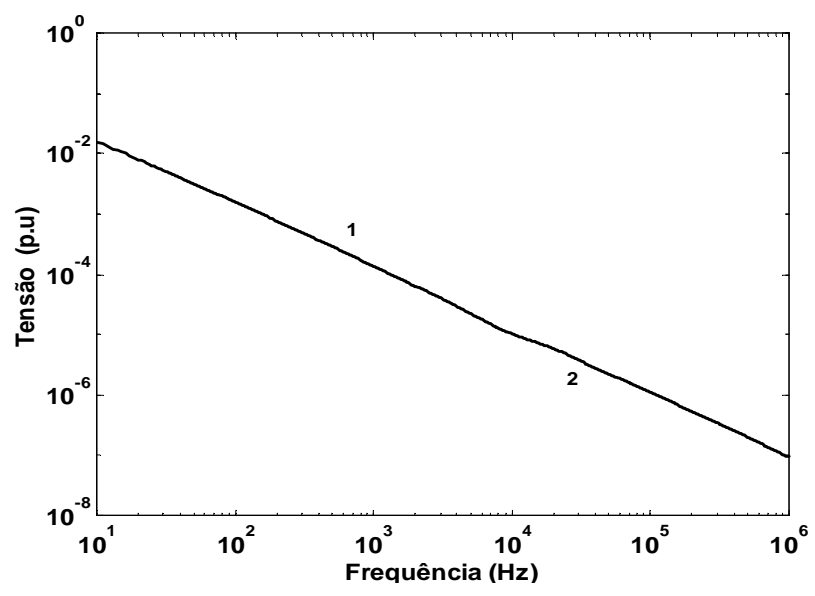

Figura 12: Módulo da tensão no terminal receptor da fase 1: Método convencional (curva 1) e método proposto (curva 2).

sendo que neste caso será dito que a linha foi separada em seus modos exatos por meio do método convencional. A curva 2 mostra resultados obtidos quando a linha é separada em seus modos exatos por meio do método proposto.

As Figuras 12 e 13 mostram que a resposta em freqüência obtida quando a linha é separada em seus modos exatos por meio do método convencional é idêntica à resposta obtida quando utiliza-se o método proposto. Portanto conclui-se que o método de decomposição modal proposto neste artigo está correto.

Neste artigo, não foi feito um estudo da matriz que desacopla as componentes $\alpha$ e zero sendo que este estudo será objetivo de trabalhos futuros.

\section{CONCLUSÕES}

Neste trabalho foi sugerido o uso de duas matrizes de transformação para decompor uma linha trifásica, com plano de simetria vertical, em seus modos exatos de propagação.

O método consiste em, inicialmente, separar a linha em suas componentes $\alpha$, $\beta$ e zero utilizando a matriz de Clarke cujos elementos são números reais, independentes da freqüência e, portanto, facilmente implementados em programas que si-

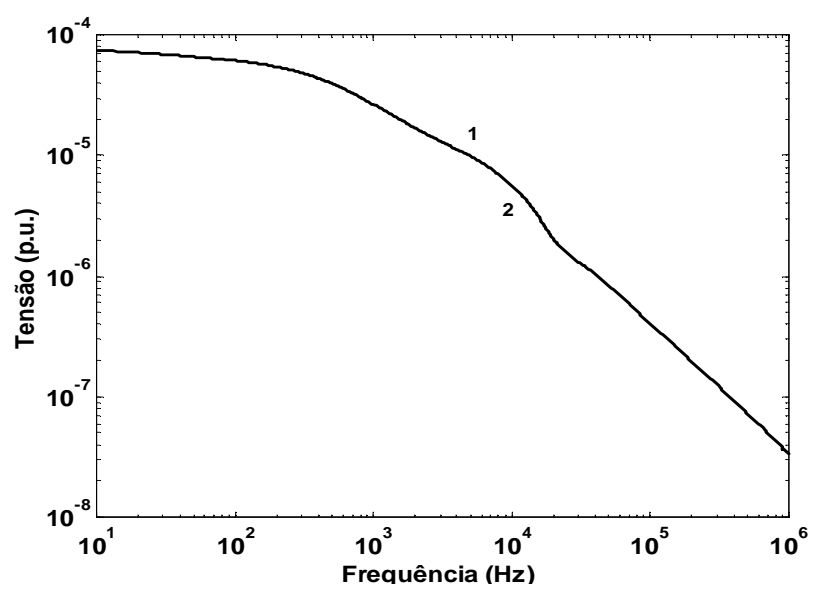

Figura 13: Módulo da tensão no terminal receptor da fase 2: Método convencional (curva 1) e método proposto (curva 2).

mulam transitórios eletromagnéticos diretamente no domínio do tempo. Em seguida as componentes mutuamente acopladas $\alpha$ e zero são tratadas como sendo uma linha bifásica, sem plano de simetria vertical, que pode ser decomposta em seus modos exatos por meio do uso de uma matriz de transformação modal adequada.

Os resultados obtidos mostraram que o método pode ser aplicado na linha trifásica de $440 \mathrm{kV}$, mostrada na Figura 6.

A utilização do método de decomposição modal proposto neste artigo pode simplificar bastante o processo de busca de uma matriz de transformação modal adequada para decompor linhas trifásicas não idealmente transpostas, mas que possuem um plano de simetria vertical, em seus modos exatos.

Esta simplificação está no fato de que uma linha trifásica, cujas fases são acopladas, é separada em uma linha monofásica e em uma linha bifásica, sendo que estas duas linhas não possuem acoplamento entre elas. Portanto, devemos nos preocupar somente com o acoplamento entre as fases da linha bifásica. Acreditamos que esta é amaior contribuição do artigo.

Novos estudos devem ser desenvolvidos para encontrar uma matriz de decomposição modal adequada para a linha bifásica mostrada na Figura 5.

\section{AGRADECIMENTOS}

Os autores agradecem ao Conselho Nacional de Desenvolvimento Científico e Tecnológico (CNPq) e à FAPESP (Fundação de Amparo à pesquisa) a concessão verbas para compra de equipamentos e de bolsas de estudos que possibilitaram o 
desenvolvimento deste trabalho.

\section{REFERÊNCIAS}

Budner, A. (1970). Introduction of Frequency-Dependent Line Parameters into an Electromagnetic Transients Program. IEEE Transactions on Power Apparatus and Systems, Vol. 89, $\mathrm{n}^{\circ}$ 1, pp 88-97.

Chen, C. T. (1984). Linear system theory and design. CBS College Publishing, New York.

Dommel, H. W. (1986). Electromagnetic Transients Program Reference Manual, Department of Electrical Engineering, University of British Columbia, Vancouver, Canada.

Marti, L. (1983). Low-Order Approximation of Transmission Line Parameters for Frequency-Dependent Models. IEEE Transactions on Power Apparatus and Systems, Vol. 102, $\mathrm{n}^{\circ}$ 11, pp 3582-3589.

Minegishi, S., H. Echigo \& R. Sato (1994). A Method for Measuring Transients Caused by Interrupting Current Using a Transmission Line Terminated in its Characteristics Impedance, IEEE Transactions on Electromagnetic Compatibility, Vol. 36, $\mathrm{n}^{\circ}$ 3, pp 244-247.

Sarto, M. S., A. Scarlatti \& C. L. Holloway (2001). On the Use of Fitting Models for the Time-Domain Analysis of Problems With Frequency-Dependent Parameters. Proc. of the 2001 IEEE Electromagnetic Compatibility International Symposium, Montreal, Canadá, pp. 588593.

Tavares, M. C, J. Pissolato \& C. M. Portela (1999). QuasiModes Multiphase Transmission Line Model. Electric Power Systems Research, Vol. 49, $\mathrm{n}^{\circ}$ 3, pp 159-167.

Tavares, M. C., J. Pissolato and C. M. Portela (1998). New Mode-Domain Multiphase Transmission line for Power Systems Studies. Proc. Of the 1998 IEEE International Symposium on Circuits and Systems, Monterey, USA.

Wedepohl, L.M., H. V. Nguyen \& G. D. Irwin (1996). Frequency-Dependent Transformation Matrices for Untransposed Transmission Lines Using NewtonRaphson Method. IEEE Transactions on Power Systems, Vol. 11, $\mathrm{n}^{\circ} 3$, pp 1538-1546. 